\title{
The flux of ultra-high energy cosmic rays after ten years of operation of the Pierre Auger Observatory
}

\author{
Inés Valiño ${ }^{* a}$ for the Pierre Auger Collaboration ${ }^{b}$ \\ ${ }^{a}$ Departamento de Física de Partículas e IGFAE, Universidade de Santiago de Compostela, \\ Spain \\ ${ }^{b}$ Observatorio Pierre Auger, Av. San Martín Norte 304, 5613 Malargüe, Argentina \\ E-mail: auger_spokespersons@fnal.gov \\ Full author list: http://www.auger.org/archive/authors_2015_06.html
}

\begin{abstract}
The flux of ultra-high energy cosmic rays has been measured with unprecedented precision at the Pierre Auger Observatory. We report an update of the all-sky flux of cosmic rays above $3 \times 10^{17} \mathrm{eV}$ obtained by combining four independent data sets. These measurements are based on data from the surface detector arrays (divided into two sets according to the shower zenith angle), from a nested, denser, detector array, and hybrid events measured simultaneously with both the fluorescence detector and the surface detector array. The spectral features are presented in detail and the systematic uncertainties are addressed. The huge amount of data collected to date, with a total exposure exceeding $50,000 \mathrm{~km}^{2} \mathrm{sr} y \mathrm{r}$, together with the wide range of sky observed (in declination from $-90^{\circ}$ to $45^{\circ}$ ) also allow us to measure the energy spectrum from different regions of the sky. We present the results of the search for a dependence of the measured flux on the declination of the incoming directions.
\end{abstract}

The 34th International Cosmic Ray Conference,

30 July- 6 August, 2015

The Hague, The Netherlands

${ }^{*}$ Speaker. 


\section{Introduction}

Over a century after their discovery, the measurement of the energy spectrum of ultra-high energy cosmic rays (UHECRs) remains as one of the main issues within the field, being fundamental to the unveiling of the origin of these particles and understanding their propagation. The Pierre Auger Observatory $[1,2]$ has collected high-quality data for more than 10 years, which has already led to a measurement of the flux of UHECRs above $3 \times 10^{17} \mathrm{eV}$ with unprecedented statistics. Two relevant spectral features have been established beyond doubt: the hardening in the spectrum at about $5 \times 10^{18} \mathrm{eV}$ (the ankle), and a strong suppression of the flux at the highest energies. The accurate measurement of the spectrum, combined with results from the study of the mass composition and of the distribution of the arrival directions of the primaries over the sky, presents a challenge for astrophysical modelling of origin and propagation of UHECRs [3].

The energy spectrum can also be exploited to study the distribution of cosmic-ray sources by searching for a flux variation with declination $(\delta)$ of the incoming directions. This study is of particular interest to the discussion of the difference seen in the suppression region between the spectra measured by Auger and by the Telescope Array (TA) experiment [4], which, despite being still compatible within the quoted systematic uncertainties of both experiments, is not understood so far. We also expect to find a $\delta$-dependence of the measured flux compatible with the hint of a dipole anisotropy for cosmic rays with energies above $8 \times 10^{18} \mathrm{eV}$ recently reported in $[5,6]$.

This paper deals with the energy spectrum of UHECRs obtained by combining the measurements of the surface detector array (SD) and the fluorescence detector (FD). The SD, spread over an area of $3000 \mathrm{~km}^{2}$, is composed of a baseline array of 1600 water-Cherenkov detectors separated by $1500 \mathrm{~m}$ in a hexagonal grid, and a smaller nested array of 49 additional detectors spaced by $750 \mathrm{~m}$ covering an area of $24 \mathrm{~km}^{2}$. The FD comprises 27 telescopes at 5 perimeter buildings viewing the atmosphere over the array. The hybrid technique developed exploits the large aperture of the SD, operating continuously, as well as the calorimetric measurement of the shower energy deposited in the atmosphere obtained with the FD which, by contrast, has duty cycle limited to clear moonless nights (13\%). This allows energy-spectrum measurements weakly reliant upon shower simulations.

\section{Measurements of the cosmic-ray energy}

The FD allows the measurement of the electromagnetic energy released by the shower in the atmosphere as a function of the atmospheric depth, $\mathrm{d} E / \mathrm{d} X$. The total primary energy is then derived by integrating this longitudinal profile over the $X$-range and adding an estimate of the so-called "invisible energy" carried into the ground by high-energy muons and neutrinos. The shower-energy estimated with the FD, $E_{\mathrm{FD}}$, has a total systematic uncertainty of 14\% [7]. The hybrid measurement is based on the selection and reconstruction of showers observed by the FD in coincidence with at least one SD station, which enables an accurate determination of the shower geometry and consequently of the energy of the primary particle. To ensure good energy reconstruction, only events that satisfy strict quality criteria are accepted [8].

The SD samples the shower particles that reach the ground. The intensities of the signals registered in the stations of the SD are used to quantify the shower size and the impact point of the shower axis on the ground. The reconstruction technique used depends upon the zenith angle $(\theta)$ 


\begin{tabular}{|c|c|c|c|c|}
\hline & \multicolumn{2}{|c|}{ SD-1500 m } & \multirow[t]{2}{*}{ SD-750 m } & \multirow[t]{2}{*}{ Hybrid } \\
\hline & vertical & inclined & & \\
\hline Data-taking period & $01 / 2004-12 / 2014$ & $01 / 2004-12 / 2013$ & $08 / 2008-12 / 2014$ & $11 / 2005-12 / 2013$ \\
\hline Exposure $\left[\mathrm{km}^{2} \mathrm{sr} \mathrm{yr}\right]$ & $42500 \pm 1300$ & $10900 \pm 300$ & $150 \pm 5$ & $1500 \pm 20$ at $10^{19} \mathrm{eV}$ \\
\hline Zenith angle [deg] & $0-60$ & $60-80$ & $0-55$ & $0-60$ \\
\hline Threshold energy & $3 \times 10^{18} \mathrm{eV}$ & $4 \times 10^{18} \mathrm{eV}$ & $3 \times 10^{17} \mathrm{eV}$ & $10^{18} \mathrm{eV}$ \\
\hline Number of events & 102901 & 15614 & 61130 & 9346 \\
\hline Number of hybrid events & 1731 & 255 & 469 & \\
\hline Energy scale (A) & $(0.1871 \pm 0.004) \mathrm{EeV}$ & $(5.71 \pm 0.09) \mathrm{EeV}$ & $(12.87 \pm 0.63) \mathrm{PeV}$ & \\
\hline Energy scale (B) & $1.023 \pm 0.006$ & $1.01 \pm 0.02$ & $1.013 \pm 0.013$ & \\
\hline Energy resolution [\%] & $15.3 \pm 0.4$ & $19 \pm 1$ & $13 \pm 1$ & \\
\hline
\end{tabular}

Table 1: Summary of the experimental parameters describing the different data sets used to measure the energy spectrum at the Pierre Auger Observatory.

of the incoming direction which defines the amount of atmosphere traversed by the shower, and therefore the level of attenuation of the shower components. We distinguish between cosmic-ray showers with $\theta<60^{\circ}$, defined as vertical events, and those with $60^{\circ}<\theta<80^{\circ}$, defined as inclined.

For vertical events, the energy estimator is the observed signal $S\left(r_{\mathrm{opt}}\right)$ at an optimal distance $r_{\text {opt }}$ from the shower axis [2]. The energy estimators are $S(1000)$ and $S(450)$ for the $1500 \mathrm{~m}$ and $750 \mathrm{~m}$ arrays respectively. For a given energy, the value of $S\left(r_{\mathrm{opt}}\right)$ decreases with $\theta$, due to the attenuation of the shower particles in the atmosphere and geometrical effects. The Constant Intensity Cut method is used to correct the energy estimator $S(1000)(S(450))$ for the $\theta$-dependence and estimate the signal $S_{38}\left(S_{35}\right)$ that the shower would have produced at the median zenith angle of $38^{\circ}$ $\left(35^{\circ}\right)$. Inclined events are reconstructed using a different procedure [9] since muons dominate the SD signals, developing asymmetric footprints at ground due to the geomagnetic field. The energy estimator, $N_{19}$, is defined as the normalisation of the muon content of a particular event relative to a reference 2D muon distribution at ground, derived from simulated proton showers with an energy of $10^{19} \mathrm{eV}$ for a given arrival direction. $N_{19}$ is thus independent of the zenith angle. To ensure a good reconstruction, only events well-contained in the SD array are selected. This fiducial trigger requires that the detector with the highest signal is enclosed in a hexagon of 6 active stations.

The absolute calibration of the SD is inferred from a high-quality subset of hybrid events used to calibrate the SD energy estimators with the calorimetric energies measured with the FD (full details in $[9,10,11])$. Only events with energies in the range of full efficiency of the SD (see values in Table 1) are used in the calibration. Here we update the SD energy scale using hybrid data up to 31 Dec 2013, increasing the data samples by about $20 \%$ with respect to those used previously. The correlations between the different $\mathrm{SD}$ energy estimators and $E_{\mathrm{FD}}$ are well described by a simple power-law function $E_{\mathrm{FD}}=A(\hat{S})^{B}$ with $\hat{S}=S_{38}, S_{35}$ or $N_{19}$. We fit this function to the selected data using a tailored maximum-likelihood method [12] (see Fig. 1). The best-fit parameters are given in Table 1. Although statistical uncertainties of the calibration constants $A$ and $B$ affect the SD energy scale, their contribution is small (at the few \% level), decreasing as the number of events increases. The main contribution to the systematic uncertainty in the SD energy scale comes from the uncertainties on $E_{\mathrm{FD}}$ that are correlated between different showers. This means that the SD shares the uncertainty of the FD energy scale of $14 \%$. 
The resolution in the SD energy is computed from the distribution of the ratio $A(\hat{S})^{B} / E_{\mathrm{FD}}$ for the hybrid events used for the calibration, assuming a fixed FD energy resolution of $7.6 \%$. The resulting average resolutions are reported in Table 1.

\section{Energy spectrum}

The final step in measuring the energy spectrum is a precise determination of the exposure for the observations. Above the energy for full detector efficiency, the calculation of the SD exposure is based solely on the determination of the geometrical aperture of the array for the corresponding zenith-angle interval and of the observation time. The choice of a fiducial trigger based on active

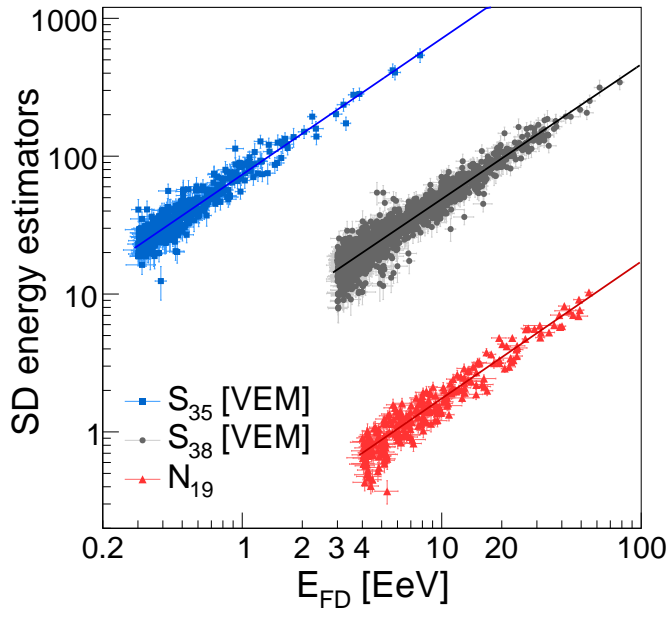

Figure 1: Correlation between the energy estimators (see text) and the energy FD energy. $S_{38}$ and $S_{35}$ are given in units of Vertical Equivalent Muon or VEM, corresponding to the signal produced by a vertical muon traversing the detector through its center. Since $N_{19}$ is a scaling factor it is dimensionless.

hexagons allows one to exploit the regularity of the array, and to compute the aperture simply as the sum of the areas of all active hexagons. The calculation of the hybrid exposure is more complex. It relies on a detailed time-dependent Monte Carlo (MC) simulation which exactly reproduces the data taking conditions and includes the response of the Hybrid detector [8]. The result is an exposure growing with shower energy above the threshold energy of $10^{18} \mathrm{eV}$.

A correction must be applied to the measured flux to account for the effect of the finite resolution in the energy determination, responsible for bin-to-bin event migration. For a steeply-falling spectrum, upward movements of reconstructed energies into a given bin are not compensated by movements from the opposite direction. The net effect is that the measured spectrum is shifted towards higher energies with respect to the true one. For the hybrid measurement, this is corrected by calculating the exposure as a function of the reconstructed energy instead of the input energy in the MC. For the SD measurements, a forward-folding approach is applied. MC simulations are used to generate a bin-to-bin migration matrix that accounts for all the resolution effects and physical fluctuations in shower development. The matrix is then used to find a flux parameterisation that fits the measured data when forward-folded, using a binned-maximum likelihood approach assuming Poisson statistics. The forward-folded spectrum is finally divided by the input flux to obtain the correction factor which is in turn applied to the measured binned spectrum to obtain the true spectrum. This correction is slightly energy dependent but is below $15 \%$ over all of the $E$-range.

Here we present an update of the measurements of the energy spectrum derived from vertical SD data sets recorded by both the $750 \mathrm{~m}$ and $1500 \mathrm{~m}$ arrays up to $31 \mathrm{Dec} 2014$, and hybrid data up to 31 Dec 2013. Moreover, we report the spectrum derived from inclined events recorded by the $1500 \mathrm{~m}$ array up to $31 \mathrm{Dec} 2013$, recently published in [13]. Values of the corresponding exposures are given in Table 1, together with other experimental parameters describing the data. Note that the exposure for the vertical SD-750 $\mathrm{m}$ data set is double the value reported previously in [14]. 

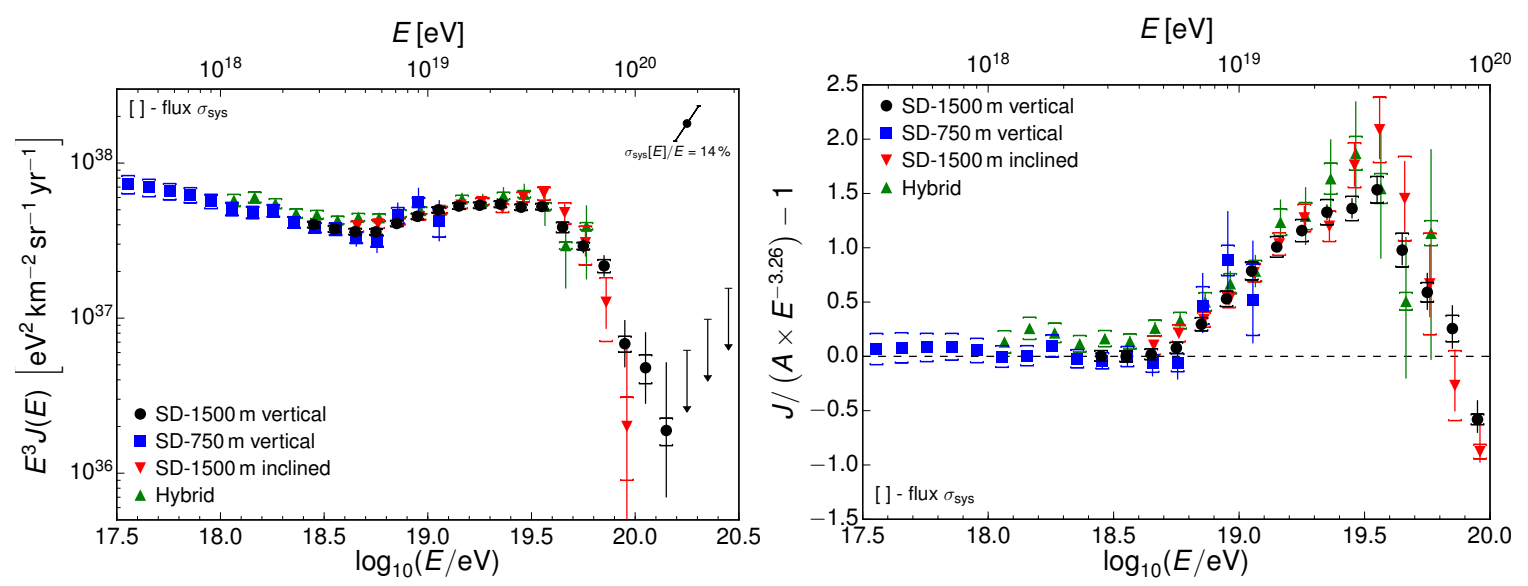

Figure 2: Left: energy spectra derived from SD and hybrid data recorded at the Pierre Auger Observatory. The error bars represent statistical uncertainties. The upper limits correspond to the $84 \%$ C.L. Right: fractional difference between the Auger spectra and a reference spectrum with an index of 3.26.

However, the number of hybrid events does not reflect the increase of exposure accumulated in 2013 due to the adoption of more stringent selection criteria from [8].

The four independent measurements of the energy spectrum of cosmic rays are shown in Fig. 2. The differential fluxes are also displayed as fractional differences with respect to a reference spectrum with an index of $3.26^{1}$. The comparison shows that all spectra are in agreement within uncertainties. The four independent measurements of the energy spectrum of cosmic rays are then combined using a method that takes into account the systematic uncertainties of the individual measurements. The systematic uncertainties of the SD-1500 vertical and inclined fluxes are 5.8\% and $5 \%$, respectively. The one of the SD-750 m flux decreases from $14 \%$ at $10^{17.5} \mathrm{eV}$ to less than $7 \%$ above $10^{18.5} \mathrm{eV}$. Similarly, the hybrid flux's uncertainty decreases from $10 \%$ at $10^{18} \mathrm{eV}$ to less than $6 \%$ above $10^{19} \mathrm{eV}$. In this procedure, the flux normalisations are used as additional constraints to derive the flux scaling factors needed to match them: $(5.7 \pm 0.2) \%$ for the vertical spectrum, $(-0.1 \pm 0.8) \%$ for the inclined spectrum, $(1.8 \pm 4.3) \%$ for the SD-750 m spectrum and $(-5.8 \pm 2.4) \%$ for the hybrid spectrum.

The characteristic features of the combined energy spectrum, shown in Fig 3, have been quantified by fitting a model that describes a spectrum by a power-law below the ankle $J(E)=$ $J_{0}\left(E / E_{\text {ankle }}\right)^{-\gamma_{1}}$ and a power-law with a smooth suppression at the highest energies:

$$
J(E)=J_{0}\left(\frac{E}{E_{\text {ankle }}}\right)^{-\gamma_{2}}\left[1+\left(\frac{E_{\text {ankle }}}{E_{\mathrm{S}}}\right)^{\Delta \gamma}\right]\left[1+\left(\frac{E}{E_{\mathrm{S}}}\right)^{\Delta \gamma}\right]^{-1} .
$$

Here, $\gamma_{1}$ and $\gamma_{2}$ are the spectral indices below and above the ankle energy $E_{\text {ankle }}$, respectively, $E_{\mathrm{s}}$ is the energy at which the differential flux falls to one-half of the value of the power-law extrapolation from the intermediate region, $\Delta \gamma$ gives the increment of the spectral index beyond the suppression region, and $J_{0}$ is the normalisation of the flux, taken as the value of the flux at $E=E_{\text {ankle. The }}$

\footnotetext{
${ }^{1}$ Reference spectrum: $J_{\text {ref }}=2.51 \times 10^{42}(E / e V)^{-3.26} \mathrm{eV}^{-1} \mathrm{~km}^{-2} \mathrm{sr}^{-1} \mathrm{yr}^{-1}$, fitted to the SD-1500 m vertical differential flux in the energy bin corresponding to $\log _{10}(E / e V)=18.55$ (bin width of 0.1 ), which contains 29371 events.
} 


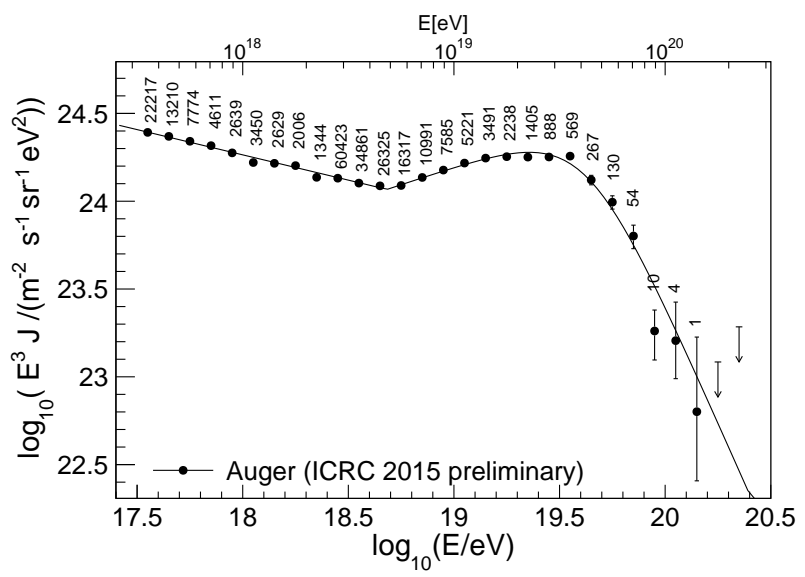

Figure 3: The combined energy spectrum of cosmic-rays as measured by the Auger Observatory, fitted with a flux model (see text). Only statistical uncertainties are shown. The systematic uncertainty on the energy scale is $14 \%$. The number of events is given above the points, which are positioned at the mean value of $\log _{10}(E / \mathrm{eV})$. The upper limits correspond to the $84 \%$ C.L.

result of the best fit is shown in Fig. 3 and the corresponding parameters are presented in Table 2, quoting both statistical and systematic uncertainties.

\begin{tabular}{cccccc}
\hline$J_{0}\left[\mathrm{eV}^{-1} \mathrm{~km}^{-2} \mathrm{sr}^{-1} \mathrm{yr}^{-1}\right]$ & $E_{\text {ankle }}[\mathrm{EeV}]$ & $E_{\mathrm{S}}[\mathrm{EeV}]$ & $\gamma_{1}$ & $\gamma_{2}$ & $\Delta \gamma$ \\
\hline$(3.30 \pm 0.15 \pm 0.20) \times 10^{-19}$ & $4.82 \pm 0.07 \pm 0.8$ & $42.09 \pm 1.7 \pm 7.61$ & $3.29 \pm 0.02 \pm 0.05$ & $2.60 \pm 0.02 \pm 0.1$ & $3.14 \pm 0.2 \pm 0.4$ \\
\hline
\end{tabular}

Table 2: Best-fit parameters, with statistical and systematic uncertainties, for the combined energy spectrum measured at the Pierre Auger Observatory.

The combined spectrum shows a flattening above the ankle, $E_{\text {ankle }}=4.8 \times 10^{18} \mathrm{eV}$, up to the onset of the flux suppression. This suppression is clearly established with a significance of more than $20 \sigma$ (the null hypothesis that the power law above the ankle continues beyond the suppression point can be rejected with such confidence). The spectral index in the region of the suppression is less certain due the low number of events and large systematic uncertainties.

A spectral observable in the GZK $[15,16]$ region that can be used to discriminate between different UHECR source-composition models is the energy $E_{1 / 2}$ at which the integral spectrum drops by a factor of two below what would be expected with no cutoff. The corresponding value derived from the Auger data, computed as the integral of the parameterisation given by eq. (3.1) with the parameters reported in Table 2, is $E_{1 / 2}=\left(2.47 \pm 0.01_{-0.34}^{+0.82}\right.$ (sys $\left.)\right) \times 10^{19} \mathrm{eV}$. This result, for instance, differs at the level of $3.4 \sigma$ from the value of $\approx 5.3 \times 10^{19} \mathrm{eV}$ predicted in [17] under the assumption that the sources of UHECRs are uniformly distributed over the universe and that they accelerate protons only. Note that, in reality, sources are discrete and in the GZK region the shape of the spectrum will be dominated by the distribution of sources around us (see [18] for example).

\section{Declination-dependence of the energy spectrum}

Given the location of the Auger Observatory at a latitude $-35.2^{\circ}$, events arriving with $\theta<60^{\circ}$ cover a wide range of declinations from $-90^{\circ}$ to $+25^{\circ}$, corresponding to a sky fraction of $71 \%$, 

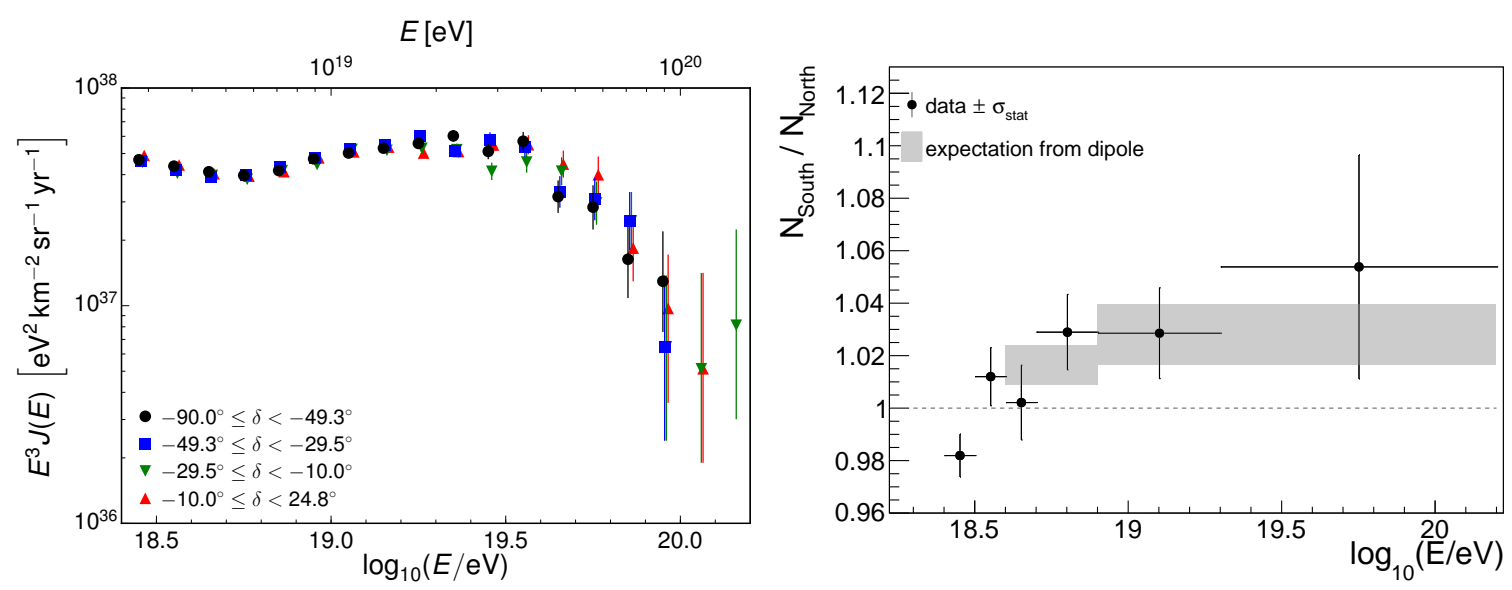

Figure 4: Left: The SD vertical energy spectrum in different declination bins. Right: the ratio of the fluxes of cosmic rays arriving from southern $\left(\delta<-29.47^{\circ}\right)$ and northern $\left(\delta>-29.47^{\circ}\right)$ directions derived from vertical events compared to the expectation from the dipolar modulation of the flux measured with Auger data with $\theta<80^{\circ}$ in the energy ranges $4<E<8 \mathrm{EeV}$ and $E>8 \mathrm{EeV}[5,6]$. The shaded boxes correspond to the propagation of the statistical uncertainties in the amplitude of the reconstructed North-South dipole component.

and therefore become an excellent data set to search for declination dependence of the measured energy spectrum. Although the inclusion of inclined events would extend the declination range to $+45^{\circ}$, only vertical showers with energy above $3 \times 10^{18} \mathrm{eV}$ are considered for this first analysis.

To search for a variation with declination of the cosmic-ray flux, it is important to carefully account for spurious effects that can modulate the flux that arise from experimental, atmospheric and geomagnetic effects. We take into account these effects following the same procedure adopted to study the large angular scale distribution of arrival directions of cosmic rays recorded at the Auger Observatory [19,5]. Firstly, the observed part of the sky is divided into four $\delta$-bands each with approximately the same exposure. The sub-spectra are shown in the left panel of Fig. 4. Given the small relative differences found between them and the all-sky spectrum $(<5 \%$ at energies below $E_{s}$ and $<13 \%$ above), there is no significant indication of a dependence on $\delta$. As a consequence, the difference seen at the highest energies (suppression region) between the spectra measured by the Auger and TA Observatories [4] can not be explained by a $\delta$-dependence of the measured flux, unless the flux measured by TA is substantially larger above declination $+25^{\circ}$ than below.

Recent studies of the distribution of arrival directions of both vertical and inclined events above $4 \times 10^{18} \mathrm{eV}$ recorded up to $31 \mathrm{Dec} 2013$ at the Auger Observatory [5, 6] have reinforced the hint of a dipole anisotropy. After performing two Rayleigh analyses in the right ascension and azimuth angles in two different energy bins, the observed amplitude in right ascension above $8 \times 10^{18} \mathrm{eV}$ suggests a large-scale anisotropy with a significance exceeding $4 \sigma$ (lower statistical significance for events with energies between 4 and $8 \mathrm{EeV})$. The reconstructed dipole points to $(\alpha, \delta)=\left(95^{\circ} \pm 13^{\circ},-39^{\circ} \pm 13^{\circ}\right)$ in the higher energy range, and $(\alpha, \delta)=\left(15^{\circ} \pm 115^{\circ},-81^{\circ} \pm 17^{\circ}\right)$ in the lower. Here we investigate if this hint is also observable in the measured flux. For this purpose, the observed sky is divided only into two bands of declination. Then the ratio of the corresponding sub-spectra is computed and compared to the expectation from this dipole anisotropy 
as shown in the right panel of Fig. 4, demonstrating good agreement between both results.

\section{Summary}

The energy spectrum above $3 \times 10^{17} \mathrm{eV}$ has been measured with unprecedented precision and statistics using the data collected by the Auger Observatory for more than 10 years. The results can be described by a power-law spectrum with spectral index 2.6 above $4.8 \times 10^{18} \mathrm{eV}$ and clearly show a steepening of the cosmic-ray flux above an energy around $4.2 \times 10^{19} \mathrm{eV}$. The dominant systematic uncertainty of the spectrum stems from the overall uncertainty in the energy scale of $14 \%$.

Differences between the recent Auger and TA spectra have motivated the search for a declination dependence of the flux of cosmic rays. No significant variation in the flux measured with the $\mathrm{SD}$ in four declination bands were found that could account for the discrepancy between spectra measured from different hemispheres. The differences found between the measurements in two separate declination bands are compatible with the variations expected from a dipolar modulation of the flux.

\section{References}

[1] The Pierre Auger Collaboration, Nucl. Instrum. Meth. A 523 (2004) 50.

[2] The Pierre Auger Collaboration, to appear in Nucl. Instrum. Meth. A, arXiv:1502.01323.

[3] A. Di Matteo, for the Pierre Auger Collaboration, paper 0249, these proceedings.

[4] Pierre Auger and Telescope Array Collaborations, I.C. Mariş et al., in Proc. of the International Symposium UHECR2014, Springdale (2014), in preparation.

[5] The Pierre Auger Collaboration, Astrophys. J. 802 (2015) 2, 111.

[6] I. Al Samarai, for the Pierre Auger Collaboration, paper 0372, these proceedings.

[7] V. Verzi, for the Pierre Auger Collaboration, in Proc. $33^{\text {rd }}$ ICRC, Rio de Janeiro (2013), arXiv:1307.5059.

[8] The Pierre Auger Collaboration, Astropart. Phys. 34 (2011) 368.

[9] The Pierre Auger Collaboration, JCAP 1408 (2014) 08, 019.

[10] R. Pesce, for the Pierre Auger Collaboration, in Proc. 32 $2^{\text {nd }}$ ICRC, Beijing, 2 (2011) 214 [arXiv:1107.4809].

[11] D. Ravignani, for the Pierre Auger Collaboration, in Proc. $33^{\text {rd }}$ ICRC, Rio de Janeiro (2013), arXiv:1307.5059.

[12] H. P. Dembinski, B. Kégl, I.C. Mariş, M. Roth, and D. Veberič, [arXiv:1503.09027].

[13] The Pierre Auger Collaboration, submitted to JCAP, arXiv:1503.07786.

[14] A. Schulz, for the Pierre Auger Collab., in Proc. 33 ${ }^{\text {rd }}$ ICRC, Rio de Janeiro (2013), arXiv:1307.5059.

[15] K. Greisen, Phys. Rev. Lett. 16 (1966) 748.

[16] G. T. Zatsepin and V. A. Kuz'min, JETP Lett. 4 (1966) 78 [Pisma Zh. Eksp. Teor. Fiz. 4 (1966) 114].

[17] V. Berezinsky, A. Z. Gazizov and S. I. Grigorieva Phys. Rev. D 74 (2006) 043005.

[18] M. Ahlers, L. A. Anchordoqui and A. M. Taylor Phys. Rev. D 87 (2013) 2, 023004.

[19] The Pierre Auger Collaboration, Astrophys. J. Suppl. 203 (2012) 34. 